\title{
Téoros
}

Revue de recherche en tourisme

\section{Le tourisme dans une région périphérique : l'exemple de l'île de Corse (France)}

\section{Gérard Richez}

Volume 13, numéro 1, mars 1994

Bas-Saint-Laurent, Gaspésie, Iles-de-la-Madeleine : fascinantes

périphéries

URI : https://id.erudit.org/iderudit/1077774ar

DOI : https://doi.org/10.7202/1077774ar

Aller au sommaire du numéro

Éditeur(s)

Université du Québec à Montréal

ISSN

0712-8657 (imprimé)

1923-2705 (numérique)

Découvrir la revue

Citer cet article

Richez, G. (1994). Le tourisme dans une région périphérique : l'exemple de l'île

de Corse (France). Téoros, 13(1), 34-37. https://doi.org/10.7202/1077774ar d'utilisation que vous pouvez consulter en ligne.

https://apropos.erudit.org/fr/usagers/politique-dutilisation/ 


\title{
Le tourisme dans une région périphérique: l'exemple de l'île de Corse (France)
}

\author{
Gérard Richez*
}

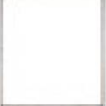

On peut avancer cinq caractéristiques principales pour introduire le sujet dans cet espace tuès particulier. II s'agit en effet de: une 1̇emontagneuse detaille moyenne; une île méditerranéenne; une île française; une île à touristes; une île à problèmes.

Quatrième île méditerranéenne par sa superficie, septième par sa population et l'une des dernières par sa densité, la Corse constitue un cas particulièrement intéressant de la problématique insulaire méditerranéenne marquée avant tout par la condition d'espace périphériqué.

\section{La Corse: une région périphérique}

En quoi et par quêlles caractéristiques la Corse est-elle une région périphérique? Quels sont les critères que l'on peut retenir comme pertinent? Et périphérique par rapport à quel centre?

Une île

La situation d'insularité implique nécessairement une couptare spatiale très forte: une distance aquatique à franchir, ce qui paraît normal puisqu'une île est définie comme un espace entouré d'eau.

Elle est donc à l'origine d'une rupture de charge nette: on doitprendre un bateau ou l'avion. Cette situation très particulière entraîne des contraintes: le respect des horaires, une attente plus ou moins longue et des pertes de temps. Le voyageur est alors soumis aux problèmes du mauvais temps qui se traduit aussi par des retards, des avions déroutés, des traversées particulièrement nauséeuses mểme avec des bateaux modernes, voire des accidents parfois graves.

La nécessité d'emprunter un ou des moyens de transports maritimes ou aériens se traduit, en outre, bien sûr, par des frais supplémentaires et des surcoúts divers. Elle entraîne une dépendance à l'égard des sociétés de transport: jusqu'en 1990, des com- pagnies Air France et Air Inter qui ont ensuite cédé la place à une compagnie aérienne régionale, la Sociêté Corse Méditerranée d'une part, et d'autre part, de la Société Nationale CorseMéditerranée (SNCM) qui bénéficie du monopole de pavillon. Cette situation est synonyme d'absence de concurrence et l'on en connaît bien les effets négatifs, et notamment le fait qu'il n'y ait pas desolution de rechange en cas de conflit social. En 1990 , une grève catastrophique de la SNCM de deux mois entraîna une pénurie de produits frais, un manque de viande et une destruction de certains produits maraîchers (salades, clémentines par exemple) qui constituent une des très rares exportations agricoles de l'île, etc., conduisant l'économie insulaire à unesituation proche de l'asphyxie. Episodiquement, des grèves des transports se produisent: quand ce ne sont pas les personnels des bateaux qui se mettent en grève, ce sont ceux des avions qui les relaient; d'autres fois ce sont les personnels des aéroports ou des ports qui en rendent l'accès impossible et tiennent l'ile en otage. $\mathrm{En} 1993$, il a fallu que la direction de la SNCM menace d'abandonner totalement ses liaisons avec le port de Marseille, déjà très gravement touché par la crise économique, pour que les dockers reviennent à la raison en acceptant la nécessaire modernisation de leur profession.

Cette situation de dépendance très forte vis-à-vis de l'extérieur a pour conséquence la nécessité de constituer des stocks tant pour les agents économiques que pour les particuliers; en outre, il est impossible de gérer l'économie avec des sflux tendus* qui caractérise la situation de très nombreuses entreprises en Europe.

La distance spatiale

La Corse est à des distances variables du continent:

- elle est très proche de l'Italie (une centaine de km) et encoreplus des íles italiennes: Elbe et la Sardaigne où 
quelques kilomètres seulement les séparent;

- la distance est plus grande avec la France: environ $220 \mathrm{~km}$ pour le trajet le plus court et de $360 \mathrm{~km}$ pour le plus long entre les ports insulaires et les deux principaux français du continent (Marseille et Nice). Au plan distance donc, une vraie périphérie comme on peut très bien le constater sur une carte.

\section{Une île montagneuse de taille moyenne}

- Sa superficie est de $8800 \mathrm{~km}^{2}$;

- elle est peu pertplée 250000 habitants en 1991, soit une densitévoisine de 30 habitants au $\mathrm{km}^{2}$, ce qui est trè̀s faible;

- une montagne omniprésente, une altitude moyenne de $568 \mathrm{~m}$ et un point culminant à $2700 \mathrm{~m}$ et des vallées nombreuses et profondes entrainant une série de difficultés diverses pour circuler dans l'espace corse. Conséquence: une multiplication des ports (7) et des aéroports (4), situation qui constitue une originalité en Méditerrannée où aucune autre île n'est dotée de tels équipements *lourdss. Cet isolement est coûteux à résorber et à entretenir; il est à la charge, pour l'essentiel, de la métropole.

\section{Une île non pas sous-développée mais non développée}

Les passibilitér agricoles limitées de la Corse jointesà une population réduite en nombre ont pour conséquences: l'existence d'un petitmarchédeconsommation; la difficulté d'écoulement de productions locales sur place qui doivent, dans le même temps, assumer le coût du transport pour exporter; une très forte dépendance vis-à-vis de l'import.

La répartition par catégorie socio-professionnelle illustre bien cette situation de non développement. Elle se caractérisepar: une hypertrophie du tertiaire qui occupe $70 \%$ des emplois; une place médiocre du secondaire: $8 \%$ pour l'industrie quasiment inexistante mais $12 \%$ pour le Bâtiment et les Travaux Publics alimentés par le tourisme, les commandes publiques et aussi par les nombreuses destructionsà l'explosif liées aux problèmes politiques, sociaux et culturels que connaît l'ille depuis près d'une vingtaine d'années; un secteur primaire relativement important: $10 \%$ et empreint d'archaïsmes qui donne parfois l'impression que lîle est devenue un *espace musé́e».

Ce type de répartition par grands secteurs d'activités économiques me paraît êttre un critère particulièrement révélateur d"une situation périphérique.

Àces caractéristiques, il faut ajouter aussi le rôle de l'économie souterraine, sans doute aussi un bon critère pour repérer une région ou un pays périphérique.

Pendant longtemps, la France n'a pas réellement cherché à mettre en valeur les potentialités de cette île non dotée, il est vrai, des éléments nécessaires, dans le passé, à mettre en place une industrie lourde (charbon, fer, etc.). La Corse fait partie des *SUDS $»$, c'est-à-dire des espaces de l'Europe méridionale restés à l'écart de la révolution industrielle et des grands courants d'échanges économiques.

Cette quasi-absence d'activités économiques se manifeste par la rareté des postes de travail qui conduisit et conduit encore nombre d'insulaires à émigrer vers la France, c'est-à-dire le centre, demandeur alors de main-d'oeuvre, qualifiée ou non, pour son industrie, son administration et son armée. Plus de 500000 Corses probablementviventettravaillentactuellement sur lecontinent. Cetteérnigration, comme partout où elle se produit, a concerné avant tout les éléments les plus dynamiques de la population, recherchant à l'extérieur un avenir meilleur qui les a, parfois, coupé de leurs compatriotesrestéssurplace. La Corse a manqué et manque enoore de cadres moyens et supérieurs capables d'impulser et d'assumer le développement.

\section{Culturellement une île bien périphérique: une forte distance psychologique}

On signalera d'abord que la Corse est française depuis relativement peu de temps: 1769 (soit 224 ans). Elle a êté achetée à la République de Gênes etn'a donc pas choisi son destin comme l'a fait la Savoie, par exemple, à la même époque.

Elle possède une langue particulière, très proche de la langue toscane, province juste en face de la côte ouest de la Corse, des traditions latines et méditerranéennes dans les styles de vie. Ses mentalités, ses relations familiales et sociales, ses traditions sont encore très fortes actuellement, même si elles tendentà s'émousser devantla culture de masse et à s'éroder rapidement face à la crise économique.

Ce particularisme entraîne la société corse dans un conflit permanent entre ce qui est externe et interne à la Corse, entre montagne et plaine, entre factions politiques. Tradition et modernité s'affrontent en permanence sur front de crise économique sévère.

\section{Un espace périphérique certes, mais} doté de forts éléments de cohésion

La langue française et la culture française sontpartout présentes et fontqueles Corses appartiennentà deux cultures. Ils disposent d'un bon niveau d'administration qui se traduit par une forte influence dans les revenus et les retraites. Le niveau de consommation et d'équipement en voitures, équipement ménager etc., est très bon. La Corse est un espace périphérique de la France, mais elle fait partie intégrante de ce pays et de son niveau économique et social que lui envient bien d'autres îles, comme les îles grecques ou les Açores portugaises.

\section{En résumé :}

- Uneillenon développée, sans avenir clair, repliée sur elle-même.

- Un espace très fortement marqué par I'insularité. L'insularité est généralement considérée, dans le contexte économique actuel, comme facteur d'entrave au développement économique et comme une source de fragilisation de l'économie. Cette opinion se trouve confortée par le fait que la plupart des îles connaissent, depuis quelques décennies, des difficultés économiques qui les ont mises en retard par rapport aux régions continentales etaux paysauxquelselles se rattachent. Les îles méditerranéennes font partie des zones défavorisées ou déprimées de ces pays tant au plan démographique qu'économique-etconnaissent de ce fait des déséquilibres importants.

- Des atouts exceptionnels, conséquences de son non développement: de l'espace, et surtout des littoraux superbes au plan paysager et quasiment vides d'activités car longtemps considérés comme inutiles et surtout dangereux en raison de la malaria. La société corse traditionnelle est une société de 
la montagne, fondée sur l'élevage, activité «noble par excellence, surtout depuis qu'elle est en cours de diminution et qu'elle a disparu totalement d'un certain nombre d'endroits.

Le tourisme de masse commence à envahir les littoraux de la France continentale dans les années soixante. Les investisseurs privés et l'Etat vont alors vite se rendre compte des atouts exceptionnels de la Corse en ce quiconcernele tourisme. Et des possibilités importantes de développement économique pour cette île pour laquelle peu de choses avaient ếté faites jusqu'alors. Le tourisme fut alors considéré dans les années soixante-dixcomme pouvant être le wlevier du développement économique de la Corses.

\section{Le tourisme en Corse : une quasi-monoactivité}

\section{Les atouts}

Ils sont nombreux: un littoral de $1000 \mathrm{~km}$ de long, soit autant que la façade méditerranéenne française continentale; un littoral superbe, varié(côte rocheusegranitique, schisteuse ou volcanique, surtout à l'ouest, sablonneuse à l'est); un littoral presque vierge d'équipements; la mer Méditerranée, belleet peupolluée par les rejetsindustriels, puisqu'il y a peu d'industries en Corse; une montagne, alpine par ses sommets déchiquetés, son relief, la présence d'un couvert neigeuxqui peutêtre important en hiver, et des névés tout l'été dans les parties les plus favorables; des forêts denses de feuillus, fraicheur estivale garantie; des torrents nombreux, donc de l'eau en relative abondance ce qui est rare en pays méditerranéen et encore plus dans les îles; des villages de montagne extrềmement pittoresques et encore habités, mais pour combien de temps?

\section{Mise en place et caractéristiques du tourisme}

\section{Les principes}

Il n'y a pas, en Corse, de plan étatique massif d'aménagement global comme cela s'est fait dans le Languedoc-Rousillon; on trouve au contraire, une multitude de projets impulsés dans leur très grande majorité de l'extérieur (capitaux privés) montrant bien que la Corse n'était qu'un support pour des opérateurs venant de l'extériẹur. Une société étatique, la Société pour l'Equipement Touristique de la Corse,
(SECTCO) fut chargée d'aiderà à miseen place d'une petite hôtellerie familiale et d'un certain nombre d'opérations destinées à promouvoir le tourisme: les résultats furent très modestes et le sleviero du développement économique de la Corse se réduisit à un fétu de paille.

\section{Les caracteristiques principales du tourisme}

Je me limiterai seulement à donner ici quelques caractéristiques très générales de cette activité.

La Corse connaît (subit?') un tourisme de masse qui atteint, bon an mal an, un million à 1,2 million de visiteurs pour une population de 250000 habitants, pour l'essentiel concentré sur l'été ( $80 \%$ environ). Le 15 août 1991, l'INSEE a estimé que 330000 touristes étaient présents sur l'ile. Venus dans leur très grande majorité en voiture, ils sont très mobiles, sillonnant l'espace corse en tous sens, donnant l'impression auxinsulaires d'unvéritableenvahissement. Adeptes du camping (*une île pour campeurs; a pu titrer un article de la revue de I'INSEE, Economie Corse) et souvent du camping *sauvages, leurs retombées économiquessont plus faibles que dansd'autres îles où l'accès en avion est essentiel (les Baléares, par exemple).

En Corse comme ailleurs, les activités touristiques se diversifient de plus en plus et se traduisent par la recherche de la qualité, de milieuxnaturelspeu transformés, d'exotisme et d'un accueil chaleureux qui fait trop souventdéfautici.Comme partout ailleurs, le touriste reste moins longtemps et surveille avec une attention très vive ses dépenses.

Enfin, en raison de l'ampleur de l'émigration insulaire et du profond attachement des Corses à leur île, on note un très fort pourcentage (le quart environ) de touristes très particuliers puisqu'il s'agit de Corses rentrant chez eux.

\section{Les mouvements}

régionalistes et nationalistes

Dans le même temps où tous ces projets fleurissaient (200 à 300000 lits dans les cartons des investisseurs pour une population qui alors n'atteignait pas 200000 habitants permanents!) et que certains voyaient le jour, la Corse des années soixante-dix, comme un certain nombre de régions françaises, a vu se développer le mouvement régionaliste, en réaction contre lecentralisme parisien. Les responsables du mouvement régionaliste puis nationaliste ont vite compris le danger, pour la culture corse, de la mise en place d'une puissante activité touristique, surtout si un certain nombre de mesures d'accompagnement n'étaient pas prises.

En effet, le tourisme, ici, a été vécu comme une agression, un néocolonialisme: les capitaux et l'essentiel du personnel venaient de l'extérieur, entraînant trop souvent une mise à l'écart des Corses.

La culture, le rythme de vie des touristes si différent, les troismois d'activités succédant à neuf mois d'inactivité étaient mal perçus par une population vieillie, repliée sur ellemême et, de ce fait, particulièrement menacée. La forte demande estivaleentrainait chaque année une hausse des prix qui ne baissaient jamais une fois la saison finie. Ajaccio et Bastia sont les deuxvilles les plus chères de France!

L'occupation des littoraux par les activités touristiques et lá spéculation qu'elle provoquea été et reste mal vécue parles Corses qui se voient ainsi dépossédés d'un patrimoine qu'ils ont, certes, eux-même contribué à dilapider.

\section{Les rejets du tourisme}

Cettesituation assez particulièresetraduisit par toute une série d'actions violentes: manifestations dégénéranten bagarres avec des blessés graves (point trop original dans la France de l'époque!), mais surtout la Corse se singularisa - et se singularise toujours - par le recours qu'elle fit aux destructions à l'explosif couplé à des bouteilles degaz, d'un certain nombre d'hôtels, de villages de vacance, de résidences secondaires appartenant à des Français originaires du continent et à des étrangers.

Mais elle entra aussi en lutte contre toutes les formes de ce qui était perçu par les nationalistes comme relevant de la domination del'Etat français: perceptions, gendarmeries, palais de justice, banques, etc., en Corse et sur le continent, notamment au travers de «nuits bleuess très spectaculaires et très médiatiques puisqu'elles regroupaient parfois plusieurs dizaines d'attentats! Certaines administrations régionales comme le siège et les bureaux du Conseil général de la Haute Corse furent aussi détruites à l'explosif en raison de leur 
rôle de «courroie» de transmission, entre le niveau national et le local et en raison du phénomène du *clanisme», considéré par les mouvements nationalistes comme étant une des origines du maldéveloppement de la Corse.

La réaction de l'administration française très centralisatrice jusqu'en 1983, date à laquelle commencent à entrer en vigueur les lois de décentralisation - fut maladroite etsouvent brutale: la répression se traduisit par l'emprisonnement de plus d'une centaine de jeunes Corses, par des forces de police omniprésentes conduisant à des *bavures» mortelles pour des innocents, par une situation quasi-insurrectionnelle, auxquelles succédèrent des périodes de calme, suivies à nouveau par des périodes de nouvelles violences selon un engrenage bien connu; les gouvernements de gauche ayant un peu moins mal gếré ce problème queceuxde droite, traditionnellement plus enclins à faire respecter la loi républicaine et le jacobisme centralisateur.

Dans le même temps, s'est développé un puissant banditisme, favorisé par la situation politique et sociale particulière issue decette période très troublée et sans doute aussi par les activités touristiques particulièrement propices aurecyclagede «l'argent sales. Ce banditisme se retrouve aussi assez souvent dans d'autres régions périphériquesinsulaires(Sardaigne, Sicile, etc).

\section{Conséquences de cette situation assez particulière}

Cette situation qui est apparue au début des année 1970 constitue un très fort handicap pour les activités économiques locales car les investisseurs possibles se détournent, bien évidemment, decette sîle à risquess.

D'où un fort ralentissement des opérations d'aménagement: quasiment toutes les grosses opérations ont été arrêtées: soit environ 200000 lits!

D'où la revente des terrains achetés à très bas prix par des Sociétés Civiles Immobilières qui renonçaient à leurs objectifs de constructions de logements touristiques. L'action du Conservatoire du littoral, organisme publicchargéd'acheter desterrains aux fins de protection et d'aménagement touristiques et récréatifs légers, s'en trouva ainsi largement stimulée.
Mais, en contrepartie, multiplication des constructions isolées, au coup par coup, sanspland'ensembleconduisant, en certains lieux, à un mitage préoccupant del'espace. Et modification dans la répartition par nationalité des touristes: baisse des Français en provenance du continent et des Allemands; et très forte augmentation des Italiens: $20 \%$ de la clientèle touristique totale et plus de la moitié des étrangers.

Et pourtant, l'actrvité touristique représente l'actrvité économique escentielle de lille. Le chiffre d'affaires a été estimé par l'INSEE à 6,6 milliards de $\mathrm{FF}$, dont 1,6 pour les transports. Et ce n'est pas le moindre paradoxe de cette île!

\section{Conclusions}

Si l'on analyse la situation de l'île de Corse au travers de trois critères majeurs qui sont la distance spatiale, l'originalité culturelle et le moindre niveau de développernent économique, la Corse est donc bien une région périphérique au même titre que d'autres ensembles insulaires français ou d'autres régions européennes. Elle fait partie de ces $\approx$ SUDS $*$, espaces en décalage important, qui bénéficient actuellement d'une attention particulière de l'Union européenne afin de tenter d'atténuer les écarts entre centre et périphérie qui sont sources de dysfonctionnements graves.

La Corse a évité, au moins en apparence, le danger du tout tourisme, d'une île livrée à unemonoactivité. Elles'estdonnéele temps de la réflexion sur ses choix de développement. Mais elle a du mal à dire clairement ce qu'elle veut et surtout à mettre en place unepolitiquecohérentededéveloppement, empêtrée qu'elle est par ses contradictions. Depuis 1981, les régions françaises - et la Corse un peu plus que les autres en raison de ses spécificités - disposent de davantage de pouvoirs. Elle devrait - elle devra - faire preuve de sa capacité à se donner les moyens d'une nouvelle politique qui devrait tendre à atténuer les effets pervers de la situation depériphérie dans laquelleelle se trouve et, au contraire, chercher à valoriser au maximum les effets positifs de se trouver en périphérie d'un centre économiquement puissant $f$ 